\title{
Cardiopulmonary exercise testing early after stroke using feedback-controlled robotics-assisted treadmill exercise: test-retest reliability and repeatability
}

\author{
Oliver Stoller ${ }^{1,2,3^{*}}$, Eling D de Bruin ${ }^{2,4,5}$, Matthias Schindelholz ${ }^{1,3}$, Corina Schuster-Amft ${ }^{1,3}$, Rob A de Bie ${ }^{2,5}$
} and Kenneth J Hunt ${ }^{1,3}$

\begin{abstract}
Background: Exercise capacity is seriously reduced after stroke. While cardiopulmonary assessment and intervention strategies have been validated for the mildly and moderately impaired populations post-stroke, there is a lack of effective concepts for stroke survivors suffering from severe motor limitations. This study investigated the test-retest reliability and repeatability of cardiopulmonary exercise testing (CPET) using feedback-controlled robotics-assisted treadmill exercise (FC-RATE) in severely motor impaired individuals early after stroke.

Methods: 20 subjects (age 44-84 years, $<6$ month post-stroke) with severe motor limitations (Functional Ambulatory Classification 0-2) were selected for consecutive constant load testing (CLT) and incremental exercise testing (IET) within a powered exoskeleton, synchronised with a treadmill and a body weight support system. A manual human-in-the-loop feedback system was used to guide individual work rate levels. Outcome variables focussed on standard cardiopulmonary performance parameters. Relative and absolute test-retest reliability were assessed by intraclass correlation coefficients (ICC), standard error of the measurement (SEM), and minimal detectable change (MDC). Mean difference, limits of agreement, and coefficient of variation (CoV) were estimated to assess repeatability.

Results: Peak performance parameters during IET yielded good to excellent relative reliability: absolute peak oxygen uptake (ICC $=0.82$ ), relative peak oxygen uptake (ICC $=0.72$ ), peak work rate (ICC $=0.91$ ), peak heart rate (ICC $=0.80)$, absolute gas exchange threshold (ICC $=0.91)$, relative gas exchange threshold (ICC $=0.88)$, oxygen cost of work (ICC $=0.87$ ), oxygen pulse at peak oxygen uptake (ICC $=0.92)$, ventilation rate versus carbon dioxide output slope (ICC =0.78). For these variables, SEM was 4-13\%, MDC 12-36\%, and CoV 0.10-0.36. CLT revealed high mean differences and insufficient test-retest reliability for all variables studied.

Conclusions: This study presents first evidence on reliability and repeatability for CPET in severely motor impaired individuals early after stroke using a feedback-controlled robotics-assisted treadmill. The results demonstrate good to excellent test-retest reliability and appropriate repeatability for the most important peak cardiopulmonary performance parameters. These findings have important implications for the design and implementation of cardiovascular exercise interventions in severely impaired populations. Future research needs to develop advanced control strategies to enable the true limit of functional exercise capacity to be reached and to further assess test-retest reliability and repeatability in larger samples.
\end{abstract}

Keywords: Stroke rehabilitation, Subacute, Severe motor impairment, Robotics-assisted gait training, Aerobic capacity, Treadmill exercise

\footnotetext{
* Correspondence: oliver.stoller@bfh.ch

'Department of Engineering and Information Technology, Institute for Rehabilitation and Performance Technology, Bern University of Applied Sciences, Burgdorf, Switzerland

${ }^{2}$ Department of Epidemiology, Maastricht University and Caphri Research School, Maastricht, The Netherlands

Full list of author information is available at the end of the article
} 


\section{Background}

Exercise capacity and activity status have become wellestablished predictors of cardiovascular and overall mortality, both of which are seriously reduced after stroke [1,2]. It has been shown that peak oxygen uptake $\left(\mathrm{VO}_{2}\right.$ peak) is approximately $50 \%$ lower compared to normative values of healthy adults 30 days post-stroke [3,4]. Despite extensive inpatient rehabilitation procedures and spontaneous recovery of cardiovascular fitness, the exercise capacity of stroke survivors entering the chronic phase remains below recommended levels [5]. The rapid deterioration of fitness not only predisposes to secondary medical complications, but also restricts the degree to which individuals can participate in rehabilitation routines and limits the ability of the individual to perform functional activities independently [6]. Therefore, research into cardiovascular exercise training in the early stages after stroke has been highlighted as a priority $[7,8]$. Effective assessment and intervention strategies are needed to assess, monitor, and improve cardiovascular fitness early after stroke.

Current research has investigated several modalities for cardiopulmonary exercise testing (CPET) in subacute stroke (6 days-6 months post-stroke) [3,4,9-13] and in chronic stroke (>6 months post-stroke) [14-19] using treadmill exercise [14-16], body weight supported treadmill exercise [3], leg cycle ergometry $[4,9-11,15,17,18]$, and combined upper- and lower-limb ergometry [12,19]. The most common concepts, i.e. treadmill exercise and leg cycle ergometry, are primarily designed for individuals with mild to moderate motor impairment, because limited motor control (non-ambulatory status, limited trunk control), poor postural control, and poor coordination of the affected limbs may restrict severely impaired individuals from performing on these devices. As a result, most studies focussing on exercise capacity after stroke have excluded individuals not able to walk independently and those presenting with low levels of motor function.

A potential option to overcome severe motor restrictions is the introduction of combined upper- and lowerlimb ergometry $[12,19,20]$. Current study results demonstrated feasibility and validity, and emphasised the fact that an all-extremity exercise protocol might decrease early onset of lower limb fatigue which leads to better estimates of exercise capacity due to the incorporation of more muscle mass. However, this approach does not embody the concept of repetitive task-specific exercise during the early stages of stroke recovery and might be not appropriate for implementation into early rehabilitation phases $[21,22]$. Considering the relatively short intervention window during subacute stroke rehabilitation and the current recommendations for cardiovascular exercise training after stroke [8], novel approaches should incorporate task-specific activities such as walking or stair climbing. The combination of motor function training and cardiovascular exercise might have the potential to positively influence overall therapy outcomes and to prevent or mitigate the loss of exercise capacity in the early stages after stroke onset [23].

A promising approach to overcome motor limitations while facilitating task-specific activity and cardiovascular stress is body weight supported treadmill training. Initial research has shown that gait symmetry improved with increasing body weight support (BWS) [24]. However, during walking with BWS of more than $15 \%$, vertical ground reaction forces and functional activity of antigravity muscles decreased, which led to substantially lower oxygen uptake levels during body weight supported treadmill training compared to conventional treadmill exercise $[25,26]$. Because severely impaired stroke survivors need considerable physical support during walking with low body weight support, the application of robotics-assisted treadmill exercise (RATE) might be of relevance in this context. A powered exoskeleton for the lower extremities, synchronised with a treadmill and BWS, provides active support during the gait trajectory that enables progressive body weight loading for individuals with severe motor restrictions.

Recent research on exercise intensity during RATE has shown substantial increases in cardiopulmonary performance parameters after stroke [27,28], and spinal cord injury [29], including complete tetraplegia [30]. However, oxygen uptake levels were below that of overground walking, recommended cardiovascular training intensities could not be achieved [31], and conventional control strategies such as the modulation of walking speed, BWS, and guidance force had only a minor influence on exercise intensity $[27,28,31]$. There is a need for voluntary effort during walking within an exoskeleton to provoke substantial cardiovascular stress comparable to conventional treadmill exercise [32]. Therefore, novel protocols have been developed to control and direct active participation during RATE with the specific aim of provoking cardiorespiratory responses [33-38]. This incorporates biofeedback mechanisms allowing the control of exercise intensity through the guidance of the individual's voluntary effort. The approach presented here provides control of exercise intensity during RATE by biofeedback and voluntary adaptation of the hip and knee forces by the subject. A first clinical study in non-ambulatory stroke survivors in the subacute phase revealed that feedback-controlled RATE (FC-RATE) can be used to implement CPET [39]. Results yielded acceptable cardiopulmonary performance parameters following standardized CPET protocols. Thus, this approach might have the potential to assess exercise capacity and guide cardiovascular exercise in stroke survivors with severe motor limitations. This needs to be formally investigated for clinical feasibility, test-retest reliability and repeatability. 
The aims of this study were: (1) to assess the clinical feasibility of FC-RATE for CPET in severely motor impaired individuals early after stroke, (2) to examine the ability of the concept to meet standard cardiopulmonary criteria for maximal exercise capacity, and (3) to assess the test-retest reliability and the repeatability of the approach.

\section{Methods}

\section{Participants}

20 first-ever stroke inpatients were recruited at a neurological rehabilitation clinic in the north-western part of Switzerland (Reha Rheinfelden) and screened according to the selection criteria. Subjects were then presented to the responsible ward physician and a cardiologist to confirm eligibility. Inclusion criteria were: (1) clinical diagnosis of initial stroke (ischemic or haemorrhagic), (2) <20 weeks after stroke onset, (3) age $>18$ years, (4) Functional Ambulation Classification (FAC) of $<3,(5)$ ability to understand the procedures and provide informed consent. Subjects were excluded if they had (1) cardiac contraindications for exercise testing according to the American College of Sports Medicine (ACSM) [40], (2) contraindications for RATE according to guidelines from the manufacturer (Hocoma AG, Volketswil, Switzerland), (3) concurrent neurological disease (e.g. Multiple Sclerosis, Parkinson's Disease, etc.), (4) concurrent pulmonary disease (e.g. COPD, etc.), (5) history of dementia.

Recorded characteristics included gender, age, body mass index, diagnosis, affected body side, time poststroke, medications, comorbidities, FAC [41] and functional independence using the Extended Barthel Index [42]. All subjects were informed about risks and benefits, and gave signed informed consent. The Ethics Review Committee of the Swiss canton of Aargau approved the study (Reference No: 2012/051).

\section{Technical implementation}

The Lokomat system (Hocoma AG, Volketswil, Switzerland) was used to implement FC-RATE. The powered exoskeleton provides control of both legs using DC motors, synchronised with an integrated treadmill $(\mathrm{h} / \mathrm{p} /$ cosmos sports \& medical GmbH, Traunstein, Germany) and a motor-driven BWS system with real time feedback control for precise body weight unloading (Lokolift, Hocoma AG). The total mechanical work rate exerted on the exoskeleton by the subject was computed from the force, moment arm and velocity data at the four active joints (hips and knees). The active mechanical work rate (Pmech), applied by the subject's effort was estimated by subtracting the passive mechanical work rate (work rate necessary to move the subject passively within the exoskeleton) from the total mechanical work rate. A manual human-in-the-loop feedback system was implemented to control the subject's active work rate. Pmech was projected onto a screen at the front of the treadmill together with a target mechanical work rate ( $P *$ mech). The subject was instructed to vary the forces applied on the exoskeleton by volitional muscle activity and to keep the measured and visualized active work rate as close as possible to the target (Figure 1).

\section{Experimental protocol}

At study entry, all included subjects completed a familiarisation session with the FC-RATE concept, which started by qualified and experienced physiotherapists adjusting the Lokomat system to provide a physiological gait pattern and to ensure that the subjects could walk comfortably. Then, an initial test of decreasing BWS continuously by $5 \%$ per minute was implemented to define the minimal possible BWS level. There was strict adherence to physiological gait pattern criteria through visual observation: (1) heel strike (physiological knee extension), (2) no foot dragging during the swing phase, and (3) active weight-bearing during the stance phase (physiological knee extension) [43]. After the first adjustments, subjects were asked to perform a short constant load exercise test for $5 \mathrm{~min}$ (P*mech $=20 \mathrm{~W}$ ) to explain the approach and practice with the feedback-control structure. Finally, the safety procedures for potential adverse events were explained in detail.

After a break of at least $24 \mathrm{~h}$, subjects then completed repeated constant load testing (CLT) and incremental exercise testing (IET) on separate days, with $48-72 \mathrm{~h}$ between the trials. All sessions were controlled for time of day. Subjects were instructed to avoid additional strenuous activity during participation in the study and not to consume food, alcohol, nicotine or caffeine at least $3 \mathrm{~h}$ prior to testing.

Subjects were asked at the beginning of the first CLT and IET to increase their maximal voluntary effort during RATE within $30 \mathrm{~s}$ to define the maximal work rate (Pmax) for the subsequent tests. Walking cadence was fixed at $60 \mathrm{steps} / \mathrm{min}$ and individual BWS was consistent for all sessions. An experienced examiner performed all tests. There was close adherence to established models for exercise testing according to the ACSM guidelines [40].

CLT was based on constant-intensity exercise (40\% Pmax) separated into 4 phases: (1) rest - subjects stood on the treadmill for 5 min with $0 \%$ BWS, (2) passive phase - subjects walked passively with their individual BWS for 5 min, (3) active phase - subjects actively contributed to the walking by pushing forward within the exoskeleton during the swing phase of each leg to reach the target work rate for $10 \mathrm{~min}$, (4) recovery - subjects walked passively with their individual BWS for $5 \mathrm{~min}$ (Figure 2A). 


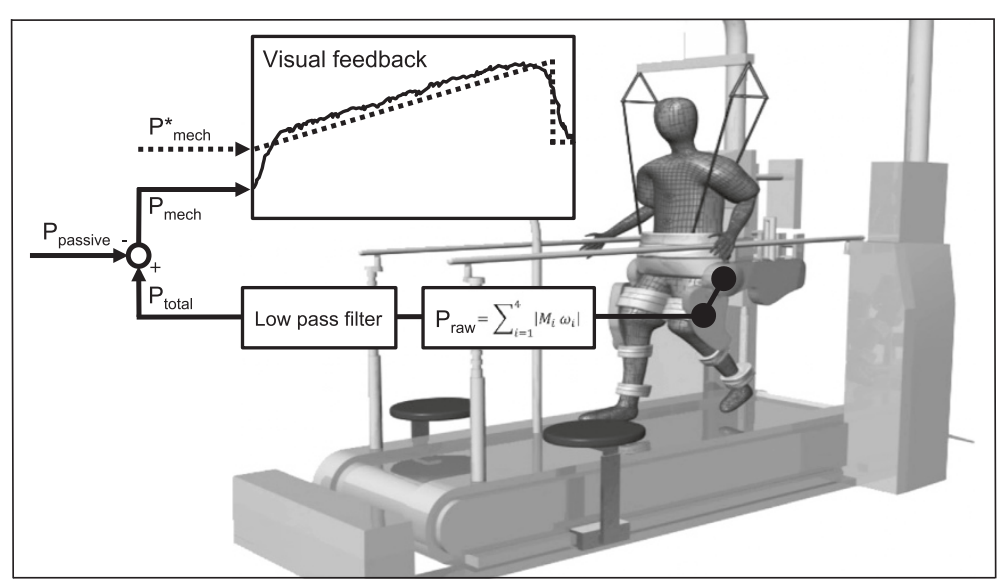

Figure 1 Feedback-controlled robotics-assisted treadmill exercise. Hip and knee joint forces and angles are measured in real time to allow calculation of the mechanical work rate (Pmech, solid line) and projection onto a screen in front of the subject. Individual target work rate profiles ( $P^{*}$ mech, dashed line) are used to guide exercise intensity during robotics-assisted walking. The passive mechanical work rate (Ppassive) is evaluated before every session and subtracted from Pmech. Legend: Praw = raw mechanical work rate, $M_{i}=$ moments of force, $\omega_{i}=$ angular velocity, Ptotal $=$ total mechanical work rate

IET was based on progressive ramp exercise and separated into 4 phases: (1) rest - subjects stood on the treadmill for 5 min with $0 \%$ BWS, (2) passive phase subjects walked passively with their individual BWS for 5 min, (3) active phase - subjects actively contributed to the walking by pushing forward within the exoskeleton during the swing phase of each leg to reach the target work rate, (4) recovery - subjects walked passively with their individual BWS for $5 \mathrm{~min}$. The progressive ramp (active phase) was defined as a continuous slope aiming to the reach predefined Pmax in 10 min (Figure 2B).

Both test protocols followed strict termination criteria for CPET including: (1) abnormal blood pressure responses, i.e. hypertensive (systolic $>210 \mathrm{mmHg} /$ diastolic $>115 \mathrm{mmHg}$ ) when exercising at high work rate, or hypotensive responses (decrease of $>10 \mathrm{mmHg}$ ) despite an increase in work rate, (2) individual work rate below target work rate for $60 \mathrm{~s}$, (3) peak heart rate within 10 beats per

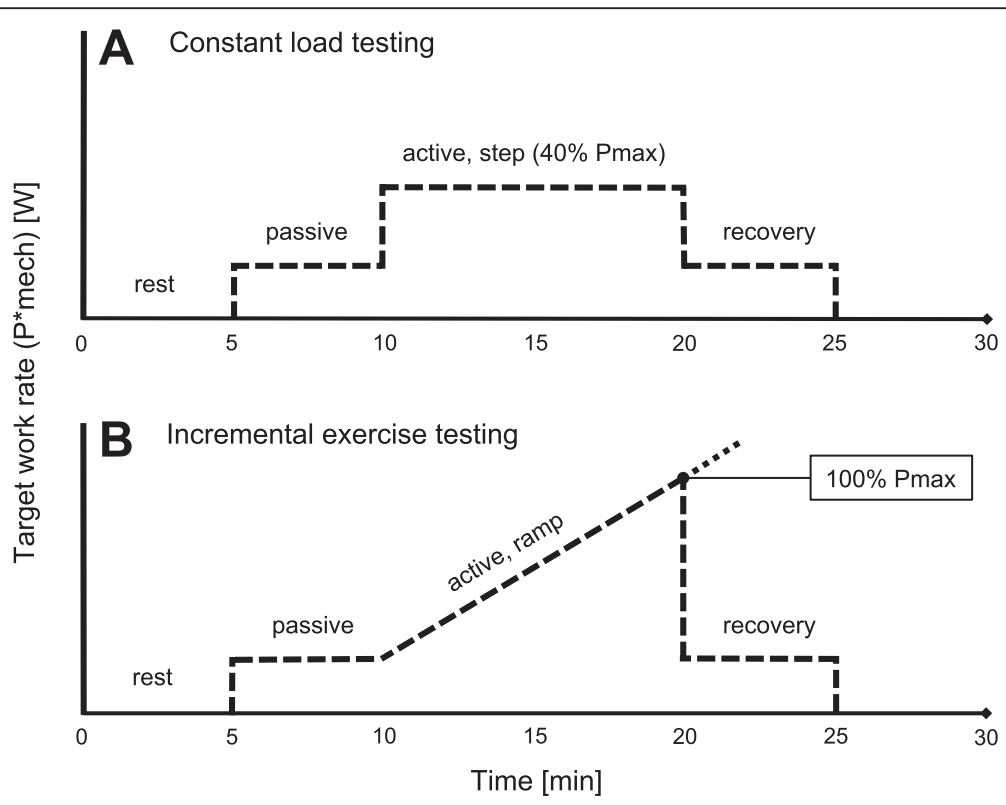

Figure 2 Exercise testing protocols. Schematic representation of constant load testing, CLT, (A) and incremental exercise testing, IET, (B) using feedback-controlled robotics-assisted treadmill exercise. The dashed line represents the target work rate ( $\mathrm{P}^{*}$ mech). The slope during incremental exercise testing was estimated such that the predefined work rate maximum (Pmax) was reached at 10 min during the active phase. When individual termination criteria were met the incremental phase was ended and $\mathrm{P}^{*}$ mech set back to the passive level (recovery). 
minute of the age-predicted heart rate maximum [44], where the formula was adjusted down to $70 \%$ of heart rate maximum for subjects on beta-blocker medications [45], (d) pain or discomfort. Subjects rated their perceived exertion using the Borg rating of perceived exertion scale $(\mathrm{RPE})(6=$ no exertion at all, $20=$ maximal exertion) [46].

Several risk management strategies were implemented to ensure subjects' safety: (1) clearly defined eligibility criteria to include medically stable subjects only, (2) screening by cardiologists to exclude subjects with potential risk factors (i.e. abnormalities in resting ECG, history of any cardiac/ cardiovascular disease, uncontrolled metabolic disease), (3) continuous blood pressure and heart rate monitoring during exercise testing, (4) presence of resuscitation-trained assistants, (5) opportunity to call the emergency medical resuscitation team in the clinic, and (6) presence of personnel trained to release the subject within $60 \mathrm{~s}$ from the exoskeleton. Detailed information on FC-RATE-based CPET can be found elsewhere $[39,47]$.

\section{Outcomes}

Measured cardiopulmonary performance parameters were: oxygen uptake $\left(\mathrm{VO}_{2}\right)$, carbon dioxide output $\left(\mathrm{VCO}_{2}\right)$, ventilation rate $\left(\mathrm{V}_{\mathrm{E}}\right)$, respiratory rate $\left(\mathrm{R}_{\mathrm{f}}\right)$, and heart rate $(\mathrm{HR})$. These were recorded by a breath-by-breath cardiorespiratory monitoring system (MetaMax 3B, Cortex Biophysik, Leipzig, Germany), including a heart rate belt (T31, Polar Electro, Kempele, Finland) and a receiver board (HRMI, Sparkfun, Boulder, USA). Pmech was calculated using the exoskeleton geometry and interaction forces, and angular signals, which were available in real time from a custom interface unit.

For CLT, outcome variables were speed of oxygen uptake kinetics (time constant $\tau$ ), oxygen cost of passive walking ( $\Delta$ rest vs. passive walking), oxygen cost of active walking ( $\Delta$ passive walking vs. active walking), and accuracy of work rate tracking $\left(\mathrm{RMSE}_{\mathrm{P}}\right)$. IET focused on peak performance parameters for oxygen uptake $\left(\mathrm{VO}_{2}\right.$ peak), time to $\mathrm{VO}_{2}$ peak $\left(\mathrm{tVO}_{2}\right.$ peak), work rate (Ppeak), ventilation rate ( $V_{\mathrm{E}}$ peak), respiratory rate $\left(\mathrm{R}_{\mathrm{fp}} \mathrm{peak}\right)$, heart rate (HRpeak), and respiratory exchange ratio (RERpeak). In addition, gas exchange threshold (GET), oxygen cost of work $\left(\Delta \mathrm{VO}_{2} /\right.$ $\Delta \mathrm{P}), \mathrm{O}_{2}$ pulse at $\mathrm{VO}_{2}$ peak $\left(\mathrm{O}_{2}\right.$ pulse $), \mathrm{V}_{\mathrm{E}}$ versus $\mathrm{VCO}_{2}$ slope $\left(\triangle \mathrm{V}_{\mathrm{E}} / \Delta \mathrm{VCO}_{2}\right)$, and $\mathrm{RMSE}_{\mathrm{P}}$ were evaluated.

\section{Data processing}

Raw breath-by-breath data were processed using a zero phase shift moving average filter over 15 breaths [48]. For CLT, the time constant for the oxygen uptake kinetics $(\tau)$ was calculated using a non-linear least-squares algorithm to fit the data as described in the following mono-exponential equation: $\mathrm{VO}_{2}(\mathrm{t})=\mathrm{VO}_{2}(\mathrm{~b})+\Delta \mathrm{VO}_{2}$ $\left(1-\mathrm{e}^{-(\mathrm{t}-\mathrm{Td}) / \mathrm{\tau}}\right), \mathrm{t}>0$, with $\mathrm{VO}_{2}(\mathrm{~b})=$ oxygen uptake at baseline, $\Delta \mathrm{VO}_{2}=$ step increase in oxygen uptake, $\mathrm{Td}=$ time delay of $20 \mathrm{~s}$ corresponding to the cardio-dynamic phase of the response, and $\tau=$ time constant [49]. Steady-state was defined by excluding the first 2 minutes and last minute of each phase, i.e. steady-state calculations were done using data from the $3^{\text {rd }}-4^{\text {th }}$ minute of a given phase. Cost of passive walking was defined as the difference between rest and passive steady-state values, whereas cost of active walking was estimated from the difference between passive and active steadystates. For IET, peak cardiopulmonary response variables were defined as the maximal values in the final $30 \mathrm{~s}$ during the incremental phase. Criteria for maximal aerobic capacity were (1) plateau in oxygen uptake, (2) respiratory exchange ratio (RER) $\geq 1.15$, and (3) peak heart rate within 10 beats per minute of the age-predicted heart rate maximum (adjusted for subjects on beta-blocker medications) [40]. The identification of a plateau or reduction in $\mathrm{VO}_{2}$ was performed by plotting the slope and 95\% confidence interval $(\mathrm{CI})$ of the $\mathrm{VO}_{2}$-Pmech slope by least-squares linear regression analysis, where the presence of data points that fell below and outside the extrapolated 95\% CI were taken as evidence of plateauing or levelling-off behaviour [50]. The GET was estimated using the v-slope method, where the anaerobic threshold is identified as the deflection point of the $\mathrm{VO}_{2}-\mathrm{VCO}_{2}$ relationship [51]. The accuracy of work rate tracking $\left(\right.$ RMSE $\left._{\mathrm{P}}\right)$ was expressed by the root mean square error between Pmech and P*mech. Data processing was performed using MATLAB (Version R2010a, MathWorks, Natick MA, USA) and LabVIEW (Version 2009, National Instruments, Austin TX, USA).

\section{Statistical analysis}

Descriptive statistics were calculated for all outcome variables. Due to the small sample size, Wilcoxon-tests were applied to exclude significant practice effects. Test-retest reliability was quantified using intraclass correlation coefficients $\left(\mathrm{ICC}_{3,1}\right)$ with $95 \% \mathrm{CI}$. The ICC provides an estimate of the relative reliability of measurement when the population under study is heterogeneous [52]. ICC results of 0.60-0.74 were considered as "good", and ICC results $>0.74$ as "excellent" [53]. Absolute reliability was determined by estimating the standard error of measurement (SEM = standard deviation of the difference (SDdiff) $\sqrt{1-\mathrm{ICC}})$ and the minimal detectable change $(\mathrm{MDC}=1.96 \times \sqrt{2} \times \mathrm{SEM})$, presented in absolute values and percentages [54,55]. Repeatability was estimated by mean difference (MD), limits of agreement (LoA) (MD $\pm 1.96 \times$ SDdiff), and coefficients of variation (CoV) (SDdiff/mean). Two-sided p-values $\mathrm{p} \leq 0.05$ were considered significant. Statistical analyses were performed using SPSS (Version 20.0, IBM, Armonk 
NY, USA) and MATLAB (Version R2010a, MathWorks, Natick MA, USA).

\section{Results}

\section{General observations}

Of the 20 subjects enrolled in the study, 1 subject showed an abnormal gait pattern due to uncontrollable spasticity during familiarisation and 1 subject developed a tibia skin lesion due to inadequate padding of the exoskeleton, which led to withdrawal from the study (Figure 3). Further, 4 withdrawals after the first IET occurred due to groin pain, lack of motivation, suspected cerebrospinal fluid leak, and acute respiratory infection. Thus, 18 subjects (90\%) performed the two CLTs and, of these, 14 (70\%) also performed the two IETs. All subjects presented with severe motor impairments and were non-ambulatory (FAC range $0-2$ ). BWS ranged between $46-77 \%$ and walking speed was set at 60 steps/minute $(0.47-0.67 \mathrm{~m} / \mathrm{s})$. The subject characteristics are summarized in Table 1.

All subjects successfully completed the predefined CPET protocol (rest, passive, active, recovery). During CLT, 3 subjects stopped the active walking phase after 5 minutes due to generalized fatigue and continued with the recovery phase. All other CLT conditions were performed according to the plan. 13 subjects completed both IETs without symptomatic responses requiring termination per safety criteria; the reason for test termination was in every case the inability to reach P*mech due to generalized and/or leg fatigue. The examiner stopped 2 consecutive IET sessions in 1 subject due to high blood pressure responses according to the safety criteria ( 2 adverse events); however, no serious adverse events occurred during testing. Mean IET duration was
$23.2 \pm 2.6 \mathrm{~min}$ (active phase: $8.2 \pm 2.6 \mathrm{~min}$ ). RPE at peak performance was $14.8 \pm 1.9$. RMSE $_{\mathrm{P}}$ values were $<10 \mathrm{~W}$.

\section{Exercise capacity}

For CLT, time constants of oxygen uptake kinetics $(\tau)$ could not be evaluated due to continuous disturbances of $\mathrm{VO}_{2}$ in the transition phases (rest/passive walking, passive walking/active walking). Cost of passive walking ( $\Delta$ rest vs. passive walking) was (mean \pm standard deviation): $\quad \mathrm{VO}_{2}=184.2 \pm 124.0 \mathrm{~mL} / \mathrm{min}, \quad \mathrm{HR}=1.4 \pm 6.4$ beats/min, and cost of active walking ( $\Delta$ passive walking vs. active walking) was: $\mathrm{VO}_{2}=45.7 \pm 56.6 \mathrm{~mL} / \mathrm{min}$, $\mathrm{HR}=2.3 \pm 3.1$ beats $/ \mathrm{min}$. RMSE $\mathrm{P}_{\mathrm{P}}$ during CLT was $5.5 \pm$ $5.5 \mathrm{~W}$. For IET, peak performance parameters were: absolute $\mathrm{VO}_{2}$ peak $=1280.9 \pm 564.8 \mathrm{~mL} / \mathrm{min}$, relative $\mathrm{VO}_{2}$ peak $=15.5 \pm 4.9 \mathrm{~mL} / \mathrm{min} / \mathrm{kg} \quad(51.6 \pm 20.5 \%$ of predicted $\mathrm{VO}_{2} \max$ [56]), $\mathrm{tVO}_{2}$ peak $=8.2 \pm 2.6 \mathrm{~min}$, Ppeak $=53.9 \pm 33.8 \mathrm{~W}, \mathrm{~V}_{\mathrm{E}}$ peak $=41.3 \pm 18.6 \mathrm{~L} / \mathrm{min}$, $\mathrm{R}_{\mathrm{f} p e a k}=36.1 \pm 8.31 / \mathrm{min}$, HRpeak $=126.0 \pm 19.5$ beats $/$ $\min (84.3 \pm 12.2 \%$ of age-predicted heart rate maximum [44]), and RERpeak $=0.92 \pm 0.09$. Absolute GET was at a $\mathrm{VO}_{2}$ of $878.9 \pm 316.6 \mathrm{~mL} / \mathrm{min}$ and relative GET at $11.0 \pm 3.1 \mathrm{~mL} / \mathrm{min} / \mathrm{kg}$, which was GET\% $=72.6 \pm 12.2 \%$ of $\mathrm{VO}_{2}$ peak. $\Delta \mathrm{VO}_{2} / \Delta \mathrm{P}$ was $20.1 \pm 14.4 \mathrm{~mL} / \mathrm{W}, \mathrm{O}_{2}$ pulse was $10.2 \pm 4.1 \mathrm{~mL} /$ beat, and $\Delta \mathrm{V}_{\mathrm{E}} / \Delta \mathrm{VCO}_{2}$ was $36.3 \pm$ 7.3 L. RMSE $E_{\mathrm{P}}$ during IET was $8.7 \pm 9.0 \mathrm{~W}$.

With respect to the 3 criteria for maximal aerobic capacity, 2 subjects (14\%) showed a plateau in $\mathrm{VO}_{2}$ at the end of IET, 1 subject (7\%) achieved an RER value $\geq 1.15$, and 5 subjects $(36 \%)$ reached peak heart rate within 10 beats per minute of the age-predicted heart rate maximum, where 2 of these subjects had an adjusted heart rate due to beta-blockers. Thus, $57 \%$ of the subjects

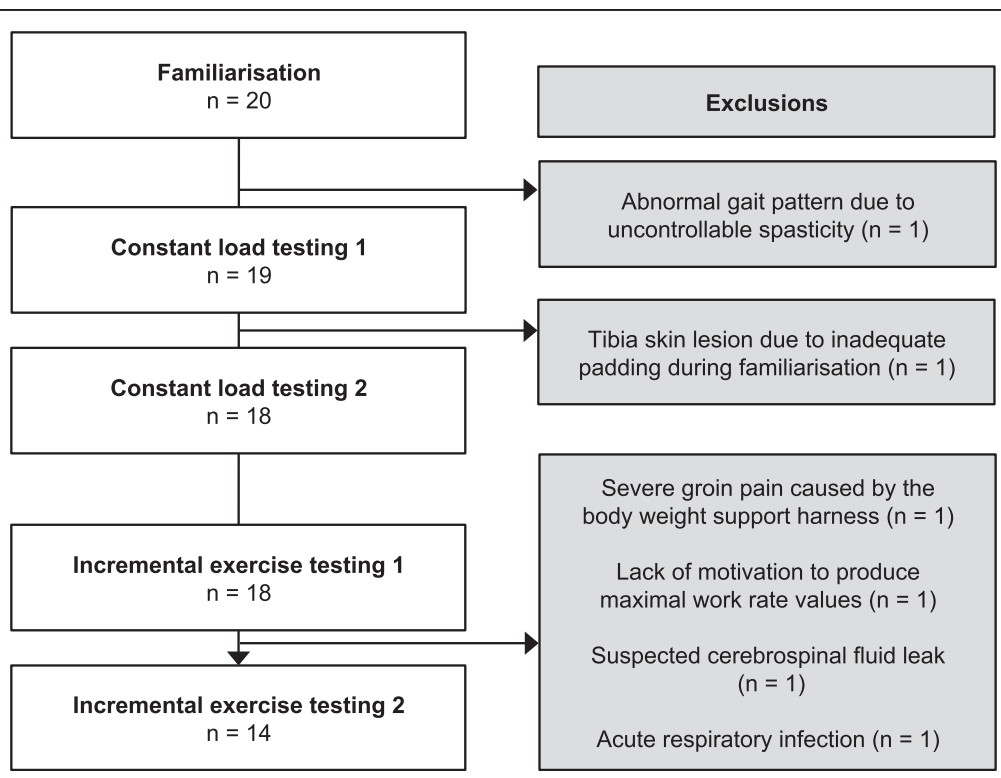

Figure 3 Study flow chart. 
Table 1 Subject characteristics

\begin{tabular}{|c|c|c|}
\hline & $\begin{array}{l}\text { Constant load testing } \\
\qquad(\mathrm{n}=18)\end{array}$ & $\begin{array}{l}\text { Incremental exercise testing } \\
\qquad(\mathrm{n}=14)\end{array}$ \\
\hline Men/women & $11 / 7$ & $9 / 5$ \\
\hline Type of stroke: ischemic/haemorrhagic & $12 / 6$ & $11 / 3$ \\
\hline Hemiparetic side: right/left & $9 / 9$ & $8 / 6$ \\
\hline Time post-stroke $[\mathrm{d}]$ & $49 \pm 31(14-139)$ & $43 \pm 25(14-92)$ \\
\hline Age $[y]$ & $61 \pm 11(44-84)$ & $61 \pm 12(44-84)$ \\
\hline BMl $\left[\mathrm{kg} / \mathrm{m}^{2}\right]$ & $27 \pm 5(19-38)$ & $28 \pm 6(19-38)$ \\
\hline FAC (0-5) & $1.1 \pm 0.8(0-2)$ & $0.9 \pm 0.8(0-2)$ \\
\hline $\mathrm{EBI}(0-64)$ & $43 \pm 9(27-56)$ & $42 \pm 9(27-55)$ \\
\hline Medications: beta-blockers/ACE inhibitors/both & $6 / 11 / 4$ & $4 / 11 / 4$ \\
\hline Comorbidities: Hypertension/Dyslipidemia/Adipositas/Diabetes mellitus & $9 / 6 / 3 / 3$ & $8 / 6 / 3 / 3$ \\
\hline BWS [\%] & $59 \pm 9(46-77)$ & $60 \pm 9(46-77)$ \\
\hline Walking speed $[\mathrm{m} / \mathrm{s}]$ & $0.57 \pm 0.05(0.47-0.67)$ & $0.56 \pm 0.05(0.47-0.64)$ \\
\hline RPE (6-20) & $13 \pm 2(6-17)$ & $15 \pm 2(11-18)$ \\
\hline Pmax [W] & $38.4 \pm 23.0(8.5-77.2)$ & $57.1 \pm 33.1(11.3-127.7)$ \\
\hline
\end{tabular}

Values are given in numbers $(\mathrm{n})$ or mean \pm standard deviation (range).

Abbreviations: BMI Body mass index, FAC Functional Ambulation Classification, EBI Extended Barthel Index, ACE inhibitors Angiotensin-converting enzyme inhibitors, $B W S$ Body weight support, RPE Rate of perceived exertion, Pmax Maximal work rate.

achieved at least 1 of the 3 criteria for maximal exercise capacity.

\section{Test-retest reliability and repeatability}

Table 2 shows mean values, test-retest reliability and repeatability results of the repeated CLT and IET trials. No practice effects could be detected; trials were not significantly different. Outcome variables for CLT yielded high MD between tests and insufficient test-retest reliability and repeatability throughout. For IET, good to excellent relative reliability was found for absolute $\mathrm{VO}_{2}$ peak ( $\left.\mathrm{ICC}=0.82\right)$, relative $\mathrm{VO}_{2}$ peak $(\mathrm{ICC}=0.72)$, Ppeak (ICC =0.91), HRpeak (ICC =0.80), absolute GET $(\mathrm{ICC}=0.91)$, relative GET $(\mathrm{ICC}=0.88), \Delta \mathrm{VO}_{2} / \Delta \mathrm{P}$ $(\mathrm{ICC}=0.87), \mathrm{O}_{2}$ pulse $(\mathrm{ICC}=0.92)$, and $\Delta \mathrm{V}_{\mathrm{E}} / \Delta \mathrm{VCO}_{2}$ $(\mathrm{ICC}=0.78)$. SEM were between $4-13 \%$ and $\mathrm{MDC}$ ranged from $12-36 \%$. MD \pm SDdiff of the outcome variables that were analysed for relative reliability were: absolute $\mathrm{VO}_{2}$ peak $=45.5 \pm 353.7 \mathrm{~mL} / \mathrm{min}$, relative $\mathrm{VO}_{2}$ peak $=1.0 \pm$ $3.8 \mathrm{~mL} / \mathrm{min} / \mathrm{kg}$, Ppeak $=2.4 \pm 15.0 \mathrm{~W}$, HRpeak $=3.6 \pm$ 12.6 beats $/ \mathrm{min}$, absolute GET $=67.3 \pm 124.2 \mathrm{~mL} / \mathrm{min}$, relative $\mathrm{GET}=0.2 \pm 1.6 \mathrm{~mL} / \mathrm{min} / \mathrm{kg}, \Delta \mathrm{VO}_{2} / \Delta \mathrm{P}=2.7 \pm$ $7.2 \mathrm{~mL} / \mathrm{min} / \mathrm{W}, \mathrm{O}_{2}$ pulse $=0.1 \pm 1.7 \mathrm{~mL} /$ beat, $\Delta \mathrm{V}_{\mathrm{E}} /$ $\Delta \mathrm{VCO}_{2}=0.5 \pm 5.1 \mathrm{~L}$. $\mathrm{CoV}$ for peak cardiopulmonary performance parameters ranged from 0.10-0.44. BlandAltman plots for the major outcome variables visualize the differences between tests (Additional files 1, 2).

\section{Discussion}

This is the first study to evaluate the test-retest reliability and repeatability of FC-RATE for assessment of exercise capacity early after severe stroke. The aims were: (1) to assess the clinical feasibility of FC-RATE for CPET in severely motor impaired individuals early after stroke, (2) to examine the ability of the concept to meet standard cardiopulmonary criteria for maximal exercise capacity, and (3) to assess the test-retest reliability and the repeatability of the approach.

\section{General observations}

Despite rigorous exclusion criteria, only $90 \%$ of the sample completed both CLTs and 70\% completed both IETs. Of the 6 subjects who dropped out during the study, only 2 were due to reasons based on uncontrollable factors such as cerebrospinal fluid leak and acute respiratory infection. The remaining 4 dropouts were caused by controllable factors such as abnormal gait pattern, tibia skin lesion, severe groin pain and lack of motivation. Skin lesions and severe groin pain due to inappropriate padding are preventable by extended familiarisation and padding procedures, whereas abnormal gait patterns due to spasticity and lack of motivation are difficult factors to control. Advanced control strategies might provide solutions for abnormal gait patterns and virtual reality approaches might facilitate motivation in the near future. Nevertheless, dropout rates were comparable with previous CPET studies in subacute stroke [3,9-11].

The guidance of work rate for FC-RATE-based CPET was successful. $\mathrm{RMSE}_{\mathrm{P}}$ values were below $10 \mathrm{~W}$, which can be seen as acceptable based on previous pilot study results [39]. The approach presented here used work rate values of both legs together and does not consider 
Table 2 Test-retest reliability and repeatability of feedback-controlled robotics-assisted treadmill exercise based cardiopulmonary exercise testing

\begin{tabular}{|c|c|c|c|c|c|c|c|c|c|c|c|c|c|c|}
\hline & \multicolumn{2}{|c|}{ Trial 1} & \multicolumn{2}{|c|}{ Trial 2} & \multirow[b]{2}{*}{$p$-value } & \multirow[b]{2}{*}{ MD } & \multirow[b]{2}{*}{ (LoA) } & \multirow[b]{2}{*}{ CoV } & \multirow[b]{2}{*}{ ICC } & \multirow[b]{2}{*}{$(95 \% \mathrm{Cl})$} & \multirow[b]{2}{*}{ SEM } & \multirow[b]{2}{*}{ SEM\% } & \multirow[b]{2}{*}{ MDC } & \multirow[b]{2}{*}{ MDCo } \\
\hline & mean $\pm S D$ & (range) & mean $\pm S D$ & (range) & & & & & & & & & & \\
\hline \multicolumn{15}{|l|}{ Constant load testing $(n=18)$} \\
\hline $\mathrm{VO}_{2}$ cost of passive walking $[\mathrm{mL} / \mathrm{min}]$ & $200.4 \pm 112.0$ & $(41.4-426.4)$ & $167.9 \pm 136.2$ & $(-3.7-506.5)$ & 0.23 & 32.5 & $(-182.4,247.5)$ & 0.58 & 0.62 & $(0.25-0.84)$ & 66.3 & 36 & 183.7 & 100 \\
\hline $\mathrm{VO}_{2}$ cost of active walking $[\mathrm{mL} / \mathrm{min} / \mathrm{W}]$ & $43.4 \pm 53.0$ & $(-2.5-212.8)$ & $48.1 \pm 61.4$ & $(2.0-214.2)$ & 0.62 & 4.6 & $(-141.2,150.5)$ & 1.59 & 0.20 & $(-0.31-0.61)$ & 65.2 & 143 & 180.8 & 395 \\
\hline Heart rate cost of passive walking [beats/min] & $0.6 \pm 5.9$ & $(-14.5-7.8)$ & $2.3 \pm 6.9$ & $(-13.0-15.4)$ & 0.68 & 1.6 & $(-13.2,16.5)$ & 5.20 & 0.33 & $(-0.15-0.68)$ & 6.1 & 426 & 16.9 & 1182 \\
\hline Heart rate cost of active walking [beats/min/W] & $2.4 \pm 3.6$ & $(0.0-15.5)$ & $2.1 \pm 2.5$ & $(0.3-9.3)$ & 0.91 & 0.3 & $(-7.1,7.6)$ & 1.62 & 0.32 & $(-0.18-0.68)$ & 3.0 & 134 & 8.4 & 370 \\
\hline Deviation of work rate $\left(\mathrm{RMSE}_{\mathrm{p}}\right)[\mathrm{W}]$ & $5.4 \pm 5.3$ & $(1.4-20.9)$ & $5.5 \pm 5.8$ & $(0.9-21.4)$ & 0.95 & 0.0 & $(-11.2,11.3)$ & 1.03 & 0.50 & $(0.05-0.78)$ & 4.0 & 73 & 11.0 & 201 \\
\hline \multicolumn{15}{|l|}{ Incremental exercise testing $(n=14)$} \\
\hline Peak $\mathrm{VO}_{2}$ uptake $\left(\mathrm{VO}_{2}\right.$ peak absolute $)[\mathrm{mL} / \mathrm{min}]$ & $1258.1 \pm 612.1$ & $(460.3-2490.3)$ & $1303.6 \pm 535.5$ & $(583.2-2427.8)$ & 0.47 & 45.5 & $(-662.0,753.0)$ & 0.28 & 0.82 & $(0.53-0.94)$ & 150.5 & 12 & 417.1 & 33 \\
\hline $\begin{array}{l}\text { Peak } \mathrm{VO}_{2} \text { uptake/body mass }\left(\mathrm{VO}_{2} \text { peak relative }\right) \\
{[\mathrm{mL} / \mathrm{min} / \mathrm{kg}]}\end{array}$ & $15.0 \pm 4.8$ & $(7.2-23.4)$ & $15.9 \pm 5.2$ & $(9.1-27.9)$ & 0.33 & 1.0 & $(-6.7,8.6)$ & 0.25 & 0.72 & $(0.33-0.89)$ & 2.0 & 13 & 5.6 & 36 \\
\hline Time to $\mathrm{VO}_{2}$ peak ( $\mathrm{tVO}_{2}$ peak) [min] & $7.5 \pm 1.7$ & $(4.9-10.8)$ & $8.8 \pm 3.2$ & $(4.1-16.9)$ & 0.14 & 1.3 & $(-4.2,6.8)$ & 0.34 & 0.39 & $(-0.09-0.74)$ & 2.2 & 26 & 6.0 & 73 \\
\hline Peak work rate (Ppeak) [W] & $52.7 \pm 33.2$ & $(11.3-107.4)$ & $55.1 \pm 35.6$ & $(7.9-101.0)$ & 0.64 & 2.4 & $(-27.7,32.5)$ & 0.28 & 0.91 & $(0.74-0.97)$ & 4.5 & 8 & 12.6 & 23 \\
\hline Peak ventilation rate $\left(V_{E}\right.$ peak) $[\mathrm{L} / \mathrm{min}]$ & $40.3 \pm 18.6$ & $(15.7-82.8)$ & $42.4 \pm 19.3$ & $(21.7-96.7)$ & 0.73 & 2.1 & $(-34.5,38.6)$ & 0.44 & 0.55 & $(0.33-0.83)$ & 12.3 & 30 & 34.1 & 82 \\
\hline Peak respiratory rate (Rfpeak) [1/min] & $36.5 \pm 9.7$ & $(22.7-54.7)$ & $35.8 \pm 7.0$ & $(23.9-44.3)$ & 0.73 & 0.7 & $(-14.0,15.4)$ & 0.20 & 0.64 & $(0.18-0.87)$ & 4.4 & 12 & 12.2 & 34 \\
\hline Peak heart rate (HRpeak) [beats/min] & $124.2 \pm 17.6$ & $(97-148)$ & $127.9 \pm 21.8$ & $(95-160)$ & 0.35 & 3.6 & $(-21.6,28.9)$ & 0.10 & 0.80 & $(0.49-0.93)$ & 5.7 & 5 & 15.8 & 13 \\
\hline Peak respiratory exchange ratio (RERpeak) & $0.91 \pm 0.05$ & $(0.84-1.00)$ & $0.93 \pm 0.11$ & $(0.79-1.21)$ & 0.55 & 0.01 & $(-0.18,0.21)$ & 0.11 & 0.39 & $(-0.18-0.76)$ & 0.08 & 8 & 0.21 & 23 \\
\hline Gas exchange threshold (GET absolute) [mL/min] & $911.3 \pm 365.1$ & $(323.8-1642.7)$ & $844.0 \pm 265.0$ & $(491.2-1255.1)$ & 0.51 & 67.3 & $(-181.0,315.6)$ & 0.14 & 0.91 & $(0.74-0.97)$ & 36.6 & 4 & 101.5 & 12 \\
\hline GET/body mass (GET relative) $[\mathrm{mL} / \mathrm{min} / \mathrm{kg}]$ & $11.1 \pm 3.1$ & $(5.1-14.7)$ & $10.8 \pm 3.1$ & $(5.3-16.6)$ & 0.65 & 0.2 & $(-2.9,3.4)$ & 0.14 & 0.88 & $(0.65-0.96)$ & 0.5 & 5 & 1.5 & 14 \\
\hline GET\% of $\mathrm{VO}_{2}$ peak (GET\%) [\%] & $75.1 \pm 11.1$ & $(59.4-94.1)$ & $69.9 \pm 13.2$ & $(49.8-92.4)$ & 0.09 & 5.2 & $(-16.3,26.8)$ & 0.15 & 0.57 & $(0.08-0.84)$ & 7.1 & 10 & 19.7 & 27 \\
\hline $\mathrm{O}_{2}$ cost of work $\left(\Delta \mathrm{VO}_{2} / \Delta \mathrm{P}\right)[\mathrm{mL} / \mathrm{min} / \mathrm{W}]$ & $18.7 \pm 13.8$ & $(5.7-51.1)$ & $21.4 \pm 15.5$ & $(4.9-60.4)$ & 0.12 & 2.7 & $(-11.7,17.1)$ & 0.36 & 0.87 & $(0.66-0.96)$ & 2.6 & 13 & 7.1 & 36 \\
\hline $\mathrm{O}_{2}$ pulse at $\mathrm{VO}_{2}$ peak ( $\mathrm{O}_{2}$ pulse) $[\mathrm{mL} /$ beat $]$ & $10.2 \pm 4.6$ & $(3.2-19.2)$ & $10.3 \pm 3.8$ & $(4.4-16.2)$ & 0.73 & 0.1 & $(-3.3,3.4)$ & 0.17 & 0.92 & $(0.78-0.98)$ & 0.5 & 5 & 1.3 & 13 \\
\hline$V_{E}$ versus $\mathrm{VCO}_{2}$ slope $\left(\Delta \mathrm{V}_{\mathrm{E}} / \Delta \mathrm{VCO}_{2}\right)[\mathrm{L}]$ & $36.6 \pm 6.9$ & $(20.4-47.6)$ & $36.1 \pm 8.0$ & $(18.4-52.2)$ & 0.93 & 0.5 & $(-9.6,10.6)$ & 0.14 & 0.78 & $(0.44-0.92)$ & 2.4 & 7 & 6.6 & 18 \\
\hline Deviation of work rate (RMSE $)$ ] [W] & $8.8 \pm 10.3$ & $(1.3-32.2)$ & $8.5 \pm 7.7$ & $(1.4-27.9)$ & 0.93 & 0.3 & $(-21.9,22.5)$ & 1.28 & 0.28 & $(-0.32-0.70)$ & 9.4 & 109 & 26.2 & 301 \\
\hline
\end{tabular}

Abbreviations: SD Standard deviation, MD Mean difference, LoA Limits of agreement, CoV Coefficients of variation, ICC Intraclass correlation coefficient

$C I$ Confidence interval, SEM Standard error of the measurement, $M D C$ Minimal detectable change. 
the severe hemiplegia of the included subjects. As a result, subjects generally tend to exercise using the unaffected side more dominantly, which led to deviations from the predefined physiological gait pattern. The powered exoskeleton allowed the subjects to remain in an acceptable movement trajectory during FC-RATE. While the approach presented here aimed to recruit as much muscle mass as possible to provoke peak exercise capacity, the imbalance of muscular activation during FCRATE might be relevant when applying the method in longitudinal training interventions, because continuous imbalance in the gait cycle might facilitate unwanted compensation patterns.

Overall, the findings present a promising method for CPET in severely motor impaired individuals after stroke, but important factors such as appropriate padding and force interactions between subject and robot must be well controlled to gain improvements towards clinical feasibility.

\section{Exercise capacity}

For CLT, the difficulty of estimating the time constants for $\mathrm{VO}_{2}$ uptake kinetics $(\tau)$ in the transitions from rest to passive walking and passive walking to active walking was due to the inherent noisiness of the breath-bybreath data and the consequent poor signal-to-noise ratio. The approach presented here seems not appropriate to provide consistent CLT outcome values for the transition phases. Sudden onset of changes in BWS and walking pattern seem to have a strong impact on individual performance levels during conventional RATE that restricts valid assessment of $\mathrm{VO}_{2}$ uptake kinetics $(\tau)$ values. Cost of passive walking was comparable with previous studies using an identical setup [27,31], whereas cost of active walking was considerably higher than previous results [39]. This difference might be caused by the inclusion of non-ambulatory subjects showing severe motor impairments (FAC 2.3 vs. FAC 1.1) in the present study.

With relative $\mathrm{VO}_{2}$ peak values of $15.5 \pm 4.9 \mathrm{~mL} / \mathrm{min} / \mathrm{kg}$ (51.6\% of predicted $\mathrm{VO}_{2} \max$ ), the results confirm that exercise capacity is seriously reduced within this group of severely motor impaired stroke survivors. Peak performance parameters during IET found in this study were slightly higher compared to previous trials using leg cycle ergometry, body weight supported treadmill training, and combined upper- and lower-limb ergometry $[3,4,9-12]$. This finding might be due to the introduction of treadmill exercise based CPET that has previously confirmed higher $\mathrm{VO}_{2}$ peak values compared to leg cycle ergometry protocols [57]. Furthermore, the feedback-control approach presented in this study might have recruited additional muscle mass that provoked higher peak values compared to body weight supported treadmill training. Considering the inclusion of individuals with serious motor impairments and the comparable peak cardiovascular performance parameters, this study opens new perspectives regarding assessment of exercise capacity early after severe stroke.

The GET values observed in the current study (\%GET $=72.6 \pm 12.2 \%$ of $\mathrm{VO}_{2}$ peak) were in the upper range compared to sedentary healthy individuals (50-76\% of $\mathrm{VO}_{2}$ peak) [58], providing additional evidence of compromised exercise capacity in this population. $\Delta \mathrm{VO}_{2} / \Delta \mathrm{P}$ was higher compared to leg cycle ergometry and conventional treadmill exercise meaning that subjects required more oxygen for a given work rate level; this may be explained by a substantial amount of unaccounted work performed during the test [59-61]. Further research seems indicated to explore the impact on $\Delta \mathrm{VO}_{2} / \Delta \mathrm{P}$ while walking within different robotics-assisted systems.

Although the current study provides first evidence for clinical feasibility of using FC-RATE for CPET and promising results regarding assessment of maximal exercise capacity, the issue remains of whether the concept is able to meet traditional criteria for true maximal capacity. Only 2 subjects within this study showed a plateau in $\mathrm{VO}_{2}$ at the end of IET, traditionally considered the primary criterion for maximal aerobic effort. This finding is in line with previous studies [3,10]. Even in healthy people, a plateau in $\mathrm{VO}_{2}$ response is not always seen during IET [62], therefore this criterion must be reconsidered for future analyses. With respect to the RER, only 1 subject achieved an RER value $\geq 1.15$, and only 3 subjects $(21 \%)$ reached $R E R \geq 1.0$. Compared to previous studies in subacute stroke which have shown mean RERpeak vales of 0.9 [4], 1.0 [3,10], 1.02 [13], 1.1 [9], the results presented here are clearly in the lower range, but not unusual in this early phase after stroke. At least 5 subjects (36\%) reached peak heart rate within 10 beats per minute of the age-predicted heart rate maximum, which is comparable with previous findings $[3,11]$. Considering these results, most of the subjects appear not to have reached their maximal aerobic capacity. The main reason might be generalized and/or leg fatigue, because 93\% of the subjects terminated the IET due to inability to maintain the target work rate, suggesting that impairments in strength, coordination, and sensorimotor control contribute to difficulties in producing high work rate levels. These findings are consistent with previous studies performing CPET in subacute stroke $[4,10]$. Advanced control strategies of powered exoskeletons, i.e. adapting the movement trajectory to the subject's needs (impairment level, hemiplegic side) and synchronising the treadmill inclination, might allow a more appropriate challenge progression to reach higher physical performance levels in this severely impaired population, and might provide closer approximations and comparisons to conventional treadmill based exercise testing procedures such as the Bruce or Balke protocols $[59,63]$. 
Although most of the subjects appear to have performed in the submaximal range, the estimation of the work rate slope using MWC-W was shown to be successful. The approach implemented was able to reach peak performance within 8-12 minutes $\left(\mathrm{tVO}_{2}\right.$ peak $\left.=8.2 \pm 2.6 \mathrm{~min}\right)$ during IET by defining the walking cadence at 60 steps/min while increasing the target work rate profile. This is an important finding for further research regarding the initial estimation of target work rate profiles for CPET in severely motor impaired populations.

Finally, considering the peak performance results and the low frequency of achieved criteria for $\mathrm{VO}_{2}$ max of this study, in combination with previous study results on peak exercise capacity in subacute stroke, we hypothesise that the guidelines postulated for healthy populations may not be realistic for determination of true exercise capacity in the early stages after stroke [64-66].

\section{Test-retest reliability and repeatability}

Most studies that examined test-retest reliability using CPET after stroke reported excellent relative reliability, whereas only Tang et al. revealed fair to good associations between trials $[10,14,15,17,18]$. The present study using a novel robotics-assisted treadmill-based method for assessment of exercise capacity revealed good to excellent relative reliability for the major peak cardiopulmonary performance parameters. There is only limited evidence so far on absolute reliability for CPET early after stroke. Compared to a previous study in chronic stroke using leg cycle ergometry that has shown SEM for relative $\mathrm{VO}_{2}$ peak of $1 \mathrm{~mL} / \mathrm{min} / \mathrm{kg}$ (6\%), our approach presented higher SEM values (2 $\mathrm{mL} / \mathrm{min} / \mathrm{kg}$ (13\%)) [15]. Likewise, a previous study that used semi-recumbent leg cycle ergometry in subacute stroke with cognitive impairments yielded considerably lower MDC values (e.g. relative $\mathrm{VO}_{2}$ peak: $0.97 \mathrm{~mL} / \mathrm{min} / \mathrm{kg}(4 \%)$ vs. $5.6 \mathrm{~mL} / \mathrm{min} / \mathrm{kg}$ (36\%)) compared to our study [18].

While studies in healthy subjects and individuals with cardiac or respiratory disease have revealed high repeatability $(\mathrm{CoV}<0.10)$ [64,67-69], the present study yielded considerably higher $\mathrm{CoV}$ for the major cardiopulmonary parameters (absolute $\mathrm{VO}_{2}$ peak $=0.28$, relative $\mathrm{VO}_{2}$ peak $=0.25$, Ppeak $=0.28$, HRpeak $=0.10$, absolute GET $=0.14$, relative $\mathrm{GET}=0.14, \Delta \mathrm{VO}_{2} / \Delta \mathrm{P}=0.36$, $\mathrm{O}_{2}$ pulse $\left.=0.17, \Delta \mathrm{V}_{\mathrm{E}} / \Delta \mathrm{VCO}_{2}=0.14\right)$.

There was visual suspicion of heteroscedasticity for absolute $\mathrm{VO}_{2}$ peak and Ppeak (Additional file 1); however, logarithmic transformation of the data (Additional file 2) did not change the relevant outcome variables, and thus appeared irrelevant fur further consideration. We hypothesise that the increase in bias along with higher work rate values is caused by large day-to-day variability in strength and/or coordinative capabilities. Although general factors that may contribute to variability in test- retest situations such as disease severity, patient instruction, time of day, testing procedure, and equipment, were well controlled in the protocol presented here, the novel approach might have led to additional confounding factors that could have influenced test-retest reliability and repeatability. A major factor was the high coordinative demand of the concept. Subjects not only had to walk (or pedal, as in earlier cycle ergometry studies); the challenge was to produce additional forces in the walking direction, where the exoskeleton restricted the movement. The results clearly indicated that the major reason for test termination was the inability to maintain $\mathrm{P}$ mech, which led to the assumption that muscular and/or coordinative fatigue was the reason for test termination. Therefore, variation might be reinforced by day-to-day variability (normally $\pm 3 \%$ [64]) and influenced by whether the test was maximal or not. This hypothesis is supported by the low RERpeak values reported in this study. More sophisticated strategies are required to reduce the load on the neuromuscular system while increasing cardiovascular stress during FC-RATE. This will possibly lead to a better approximation of true exercise capacity early after stroke and might improve the reliability and the repeatability of FC-RATE based CPET.

The approach presented here seems suitable for comparison of groups of stroke individuals or for assessment of group intervention effects in future studies, considering the range of between-group improvement in $\mathrm{VO}_{2}$ peak of 12.6-34.8\% [70-73] and Ppeak of 23.4-176.9\% [72-74] after cardiovascular exercise in subacute stroke. Whether the absolute reliability and the repeatability reported are adequate to identify effectiveness of intervention programmes to improve exercise capacity should be part of future studies including larger sample sizes.

\section{Limitations}

The major limitation of the current study is the small sample size, which may render the results underpowered. A sample size of at least 50 is generally seen as adequate for the assessment of the agreement parameter, based on a general guideline by Altman [75]. Considering the experimental approach of the method and the difficulty of implementing and performing CPET in the early stages after severe stroke, our sample of 20 subjects at onset was a realistic group size to evaluate first estimates from a clinical perspective.

The conventional sequencing of the test situations (CLT, CLT, IET, IET) might have led to practice effects. The severely impaired and early-post-stroke status of the individuals included in this experimental approach may justify this order to progressively increase the exercise intensity over time to control potential risks.

The present study protocol did not include ECG monitoring for reasons of practicability, which influenced the 
study sample by excluding individuals with cardiac risk factors for CPET. While around $75 \%$ of stroke survivors present some degree of cardiovascular disease [76], the sample of this study might not be representative. Nevertheless, there was an uncontrolled risk for cardiac events due to the absence of ECG despite the adherence to strict exclusion criteria for cardiovascular disease.

While the study protocol strictly controlled time of day for CPET, the tests were performed within $48 \mathrm{~h}$ or $72 \mathrm{~h}$ due to practical reasons. This time difference might have affected the recovery phase of the subjects, thus influencing the results.

\section{Conclusion}

This study presents first evidence on reliability and repeatability for CPET in severely motor impaired individuals early after stroke using a feedback-controlled roboticsassisted treadmill. The results demonstrate good to excellent test-retest reliability and appropriate repeatability for the most important peak cardiopulmonary performance parameters. These findings have important implications for the design and implementation of cardiovascular exercise interventions in severely impaired populations. Future research needs to develop advanced control strategies to enable the true limit of functional exercise capacity to be reached and to further assess test-retest reliability and repeatability in larger samples.

\section{Additional files}

Additional file 1: Bland-Altman plots. The difference between trial 2 (T2) and trial 1 (T1) is plotted against the mean of T1 and T2 for the major outcome variables.

Additional file 2: Bland-Altman plots (logarithmically transformed). The difference between trial 2 (T2) and trial 1 (T1) is plotted against the mean of $\mathrm{T} 1$ and $\mathrm{T} 2$ for the major outcome variables.

\section{Abbreviations}

ACSM: American College of Sports Medicine; BWS: Body weight support; $\mathrm{Cl}$ : Confidence interval; CLT: Constant load testing; CoV: Coefficient of variation; CPET: Cardiopulmonary exercise testing; FAC: Functional ambulation classification; FC-RATE: Feedback-controlled robotics-assisted treadmill exercise: GET: Gas exchange threshold; HR: Heart rate; ICC: Intraclass correlation coefficient; IET: Incremental exercise testing; LoA: Limits of agreement; MD: Mean difference; MDC: Minimal detectable change; $\mathrm{O}_{2}$ pulse: $\mathrm{O}_{2}$ pulse at $V_{2}$ peak; Pmax: Maximal work rate; Pmech: Work rate; P*mech: Target work rate; RATE: Robotics-assisted treadmill exercise; RER: Respiratory exchange ratio; $R_{f}$. Respiratory rate; RMSEp: Accuracy of work rate tracking (root-mean-square error); RPE: Rating of perceived exertion; SDdiff: Standard deviation of the difference; SEM: Standard error of the measurement; $\mathrm{VCO}_{2}$ : Carbon dioxide output; $\mathrm{V}_{\mathrm{E}}$ : Ventilation rate; $\mathrm{VO}_{2}$ : Oxygen uptake; $\mathrm{VO}_{2}$ peak: Peak oxygen uptake; $\Delta \mathrm{V}_{\mathrm{E}} / \Delta \mathrm{VCO}_{2}: \mathrm{VE}$ versus $\mathrm{VCO}_{2}$ slope; $\Delta \mathrm{VO}_{2} / \Delta \mathrm{P}$ : Oxygen cost of work.

\section{Competing interests}

The authors declare that they have no competing interests.

\section{Authors' contributions}

$\mathrm{OS}, \mathrm{EDB}, \mathrm{RDB}$ and $\mathrm{KH}$ were responsible for the design and the methodology of the study. OS and CSA prepared and obtained the ethical approval. MS was responsible for the technical development and maintenance and supported data processing. OS screened the subjects, performed the tests, processed and analysed the data and wrote the manuscript. EDB, RDB and $\mathrm{KH}$ supervised the process and provided expertise. EDB, MS, CSA, RDB and KH critically revised the manuscript. All authors read and approved the final manuscript.

\section{Acknowledgements}

The authors would like to acknowledge Prof. Dr. T. Ettlin, Dr. N. Urscheler, Dr. B. Spoendlin, Dr. A. Rohner, and Dr. M. Kummer for clinical support and medical advice, H. Rosemeyer, N. Springinsfeld, and D. Vosseler for assistance during recruitment and exercise testing, and Dr. J. Wandel and Dr. D. Bättig for statistical support.

\section{Author details}

${ }^{1}$ Department of Engineering and Information Technology, Institute for Rehabilitation and Performance Technology, Bern University of Applied Sciences, Burgdorf, Switzerland. ${ }^{2}$ Department of Epidemiology, Maastricht University and Caphri Research School, Maastricht, The Netherlands. ${ }^{3}$ Research Department, Reha Rheinfelden, Rheinfelden, Switzerland. ${ }^{4}$ Department of Health Sciences and Technology, Institute of Human Movement Sciences and Sport, ETH Zurich, Zurich, Switzerland. ${ }^{5}$ Centre for Evidence Based Physiotherapy, Maastricht University, Maastricht, The Netherlands.

Received: 4 June 2014 Accepted: 3 October 2014

Published: 11 October 2014

\section{References}

1. Myers J, Prakash M, Froelicher V, Do D, Partington S, Atwood JE: Exercise capacity and mortality among men referred for exercise testing. $N$ Engl J Med 2002, 346(11):793-801.

2. Paffenbarger RS Jr, Hyde RT, Wing AL, Lee IM, Jung DL, Kampert JB: The association of changes in physical-activity level and other lifestyle characteristics with mortality among men. N Engl J Med 1993, 328(8):538-545.

3. Mackay-Lyons MJ, Makrides L: Exercise capacity early after stroke. Arch Phys Med Rehabil 2002, 83(12):1697-1702.

4. Kelly JO, Kilbreath SL, Davis GM, Zeman B, Raymond J: Cardiorespiratory fitness and walking ability in subacute stroke patients. Arch Phys Med Rehabil 2003, 84(12):1780-1785.

5. MacKay-Lyons MJ, Makrides L: Longitudinal changes in exercise capacity after stroke. Arch Phys Med Rehabil 2004, 85(10):1608-1612.

6. Roth EJ: Heart disease in patients with stroke. Part 2: Impact and implications for rehabilitation. Arch Phys Med Rehabil 1994, 75(1):94-101.

7. Saunders DH, Greig CA, Young A, Mead GE: Physical fitness training for patients with stroke an updated review. Stroke 2010, 41(3):E160-E161.

8. Billinger SA, Arena R, Bernhardt J, Eng JJ, Franklin BA, Johnson CM, MackayLyons M, Macko RF, Mead GE, Roth EJ, Shaughnessy M, Tang A, American Heart Association Stroke, Council and Council on, Cardiovascular and Stroke, Nursing and Council on, Lifestyle and Cardiometabolic, Health and Council on, Epidemiology and Prevention and Council on Clinical, Cardiology: Physical activity and exercise recommendations for stroke survivors: a statement for healthcare professionals from the American Heart Association/American Stroke Association. Stroke 2014, 45(8):2532-2553.

9. Chen JK, Chen TW, Chen CH, Huang MH: Preliminary study of exercise capacity in post-acute stroke survivors. Kaohsiung J Med Sci 2010, 26(4):175-181.

10. Tang A, Sibley KM, Thomas SG, Mcllroy WE, Brooks D: Maximal exercise test results in subacute stroke. Arch Phys Med Rehabil 2006, 87(8):1100-1105.

11. Yates JS, Studenski S, Gollub S, Whitman R, Perera S, Lai SM, Duncan PW: Bicycle ergometry in subacute-stroke survivors: feasibility, safety, and exercise performance. J Aging Phys Act 2004, 12(1):64-74.

12. Hill DC, Ethans KA, MacLeod DA, Harrison ER, Matheson JE: Exercise stress testing in subacute stroke patients using a combined upper- and lower-limb ergometer. Arch Phys Med Rehabil 2005, 86(9):1860-1866.

13. Baert I, Daly D, Dejaeger E, Vanroy C, Vanlandewijck Y, Feys H: Evolution of cardiorespiratory fitness after stroke: a 1-year follow-up study, influence of pre-stroke patients' characteristics and stroke-related factors. Cerebrovasc Dis 2011, 31:189.

14. Dobrovolny CL, Ivey FM, Rogers MA, Sorkin JD, Macko RF: Reliability of treadmill exercise testing in older patients with chronic hemiparetic stroke. Arch Phys Med Rehabil 2003, 84(9):1308-1312. 
15. Eng JJ, Dawson AS, Chu KS: Submaximal exercise in persons with stroke: test-retest reliability and concurrent validity with maximal oxygen consumption. Arch Phys Med Rehabil 2004, 85(1):113-118.

16. Macko RF, Katzel LI, Yataco A, Tretter LD, DeSouza CA, Dengel DR, Smith GV, Silver $\mathrm{KH}$ : Low-velocity graded treadmill stress testing in hemiparetic stroke patients. Stroke 1997, 28(5):988-992.

17. Lennon OC, Denis RS, Grace N, Blake C: Feasibility, criterion validity and retest reliability of exercise testing using the Astrand-rhyming test protocol with an adaptive ergometer in stroke patients. Disabil Rehabil 2012, 34(14):1149-1156.

18. Olivier C, Dore J, Blanchet S, Brooks D, Richards CL, Martel G, Robitaille NM, Maltais DB: Maximal cardiorespiratory fitness testing in individuals with chronic stroke with cognitive impairment: practice test effects and test-retest reliability. Arch Phys Med Rehabil 2013, 94(11):2277-2282.

19. Billinger SA, Tseng BY, Kluding PM: Modified total-body recumbent stepper exercise test for assessing peak oxygen consumption in people with chronic stroke. Phys Ther 2008, 88(10):1188-1195.

20. Durstine $L$, Moore G, Painter P, Roberts S: ACSM's Exercise Management for Persons With Chronic Diseases and Disabilities. 3rd edition. Champaign, IL: Human Kinetics; 2009.

21. French B, Thomas LH, Leathley MJ, Sutton CJ, McAdam J, Forster A, Langhorne P, Price CIM, Walker A, Watkins CL: Repetitive task training for improving functional ability after stroke. Cochrane Database Syst Rev 2007, (4).

22. Langhorne $P$, Coupar F, Pollock A: Motor recovery after stroke: a systematic review. Lancet Neurol 2009, 8(8):741-754.

23. Mackay-Lyons M, McDonald A, Matheson J, Eskes G, Klus MA: Dual effects of body-weight supported treadmill training on cardiovascular fitness and walking ability early after stroke: a randomized controlled trial. Neurorehabil Neural Repair 2013, 27(7):644-653.

24. Hesse S, Bertelt C, Jahnke MT, Schaffrin A, Baake P, Malezic M, Mauritz KH: Treadmill training with partial body weight support compared with physiotherapy in nonambulatory hemiparetic patients. Stroke 1995, 26(6):976-981

25. Danielsson A, Sunnerhagen KS: Oxygen consumption during treadmill walking with and without body weight support in patients with hemiparesis after stroke and in healthy subjects. Arch Phys Med Rehabil 2000, 81(7):953-957.

26. MacKay M, Makrides L, Speth S: Effect of $15 \%$ body weight support on exercise capacity of adults without impairments. Phys Ther 2001, 81(11):1790-1800.

27. Krewer C, Müller F, Husemann B, Heller S, Quintern J, Koenig E: The influence of different Lokomat walking conditions on the energy expenditure of hemiparetic patients and healthy subjects. Gait Posture 2007, 26(3):372-377.

28. Stoller O, de Bruin ED, Schindelholz M, Schuster C, de Bie RA, Hunt KJ: Evaluation of exercise capacity after severe stroke using roboticsassisted treadmill exercise: a proof-of-concept study. Technol Health Care 2013, 21(2):157-166.

29. Hornby TG, Campbell DD, Zemon DH, Kahn JH: Clinical and quantitative evaluation of robotic-assisted treadmill walking to retrain ambulation after spinal cord injury. Topics in Spinal Cord Injury Rehabilitation 2005, 11(2):1-17.

30. Nash MS, Jacobs PL, Johnson BM, Field-Fote E: Metabolic and cardiac responses to robotic-assisted locomotion in motor-complete tetraplegia: a case report. J Spinal Cord Med 2004, 27(1):78-82.

31. van Nunen MP, Gerrits KH, de Haan A, Janssen TW: Exercise intensity of robot-assisted walking versus overground walking in nonambulatory stroke patients. J Rehabil Res Dev 2012, 49(10):1537-1546.

32. Israel JF, Campbell DD, Kahn JH, Hornby TG: Metabolic costs and muscle activity patterns during robotic- and therapist-assisted treadmill walking in individuals with incomplete spinal cord injury. Phys Ther 2006, 86(11):1466-1478.

33. Hunt KJ, Jack LP, Pennycott A, Perret C, Baumberger M, Kakebeeke TH: Control of work rate-driven exercise facilitates cardiopulmonary training and assessment during robot-assisted gait in incomplete spinal cord injury. Biomedical Signal Processing and Control 2008, 3(1):19-28.

34. Koenig A, Omlin X, Bergmann J, Zimmerli L, Bolliger M, Muller F, Riener R: Controlling patient participation during robot-assisted gait training. J Neuroeng Rehabil 2011, 8:14.

35. Pennycott A, Hunt KJ, Jack LP, Perret C, Kakebeeke TH: Estimation and volitional feedback control of active work rate during robot-assisted gait. Control Eng Pract 2008, 17(2):322-328.
36. Lam T, Pauhl K, Krassioukov A, Eng JJ: Using robot-applied resistance to augment body-weight-supported treadmill training in an individual with incomplete spinal cord injury. Phys Ther 2011, 91(1):143-151.

37. Duschau-Wicke A, Caprez A, Riener R: Patient-cooperative control increases active participation of individuals with $\mathrm{SCl}$ during robot-aided gait training. J Neuroeng Rehabil 2010, 7:43.

38. Banz R, Bolliger M, Muller S, Santelli C, Riener R: A method of estimating the degree of active participation during stepping in a driven gait orthosis based on actuator force profile matching. IEEE Trans Neural Syst Rehabil Eng 2009, 17(1):15-22.

39. Stoller O, Schindelholz M, Bichsel L, Schuster C, de Bie RA, de Bruin ED, Hunt KJ: Feedback-controlled robotics-assisted treadmill exercise to assess and influence aerobic capacity early after stroke: a proof-of-concept study. Disabil Rehabil Assist Technol 2013. doi:10.3109/17483107.2013.785038.

40. Thompson WR, Gordon NF, Pescatello LS: American College of Sports Medicine. Guidelines for Exercise Testing and Prescription. Eight ednth edition. Philadelphia: Lippincott Williams \& Wilkins; 2010.

41. Collen FM, Wade DT, Bradshaw CM: Mobility after stroke: reliability of measures of impairment and disability. Int Disabil Stud 1990, 12(1):6-9.

42. Collin C, Wade DT, Davies S, Horne V: The Barthel ADL index: a reliability study. Int Disabil Stud 1988, 10(2):61-63.

43. Perry J: Gait Analysis: Normal and Pathological Function. Thorofare, NJ: SLACK incorporated; 1992

44. Tanaka H, Monahan KD, Seals DR: Age-predicted maximal heart rate revisited. J Am Coll Cardiol 2001, 37(1):153-156.

45. Tesch PA: Exercise performance and beta-blockade. Sports Med 1985, 2(6):389-412.

46. Borg GA: Perceived exertion. Exerc Sport Sci Rev 1974, 2:131-153.

47. Schindelholz M, Stoller O, Hunt KJ: A software module for cardiovascular rehabilitation in robotics-assisted treadmill exercise. Biomed Signal Process Control 2014, 10:296-307.

48. Robergs RA, Dwyer D, Astorino T: Recommendations for improved data processing from expired gas analysis indirect calorimetry. Sports Med 2010, 40(2):95-111.

49. Whipp BJ, Wasserman K: Oxygen-uptake kinetics for various intensities of constant-load work. J Appl Physiol 1972, 33(3):351-356.

50. Poole DC, Wilkerson DP, Jones AM: Validity of criteria for establishing maximal $\mathrm{O} 2$ uptake during ramp exercise tests. Eur J Appl Physiol 2008 102(4):403-410.

51. Beaver WL, Wasserman K, Whipp BJ: A new method for detecting anaerobic threshold by gas-exchange. J Appl Physiol 1986, 60(6):2020-2027.

52. Streiner DL: Diagnosing tests: using and misusing diagnostic and screening tests. J Pers Assess 2003, 81(3):209-219.

53. Streiner DL, Norman GR: Health Measurement Scales: A Practical Guide to Their Development and use. Oxford, NY: Oxford University Press; 2008.

54. Weir JP: Quantifying test-retest reliability using the intraclass correlation coefficient and the SEM. J Strength Cond Res 2005, 19(1):231-240.

55. Haley SM, Fragala-Pinkham MA: Interpreting change scores of tests and measures used in physical therapy. Phys Ther 2006, 86(5):735-743.

56. Jurca R, Jackson AS, LaMonte MJ, Morrow JR, Blair SN, Wareham NJ, Haskel WL, van Mechelen W, Church TS, Jakicic JM, Laukkanen R: Assessing cardiorespiratory fitness without performing exercise testing. Am J Prev Med 2005, 29(3):185-193.

57. McKay GA, Banister EW: A comparison of maximum oxygen uptake determination by bicycle ergometry at various pedaling frequencies and by treadmill running at various speeds. Eur J Appl Physiol Occup Physiol 1976, 35(3):191-200.

58. Davis JA, Storer TW, Caiozzo VJ: Prediction of normal values for lactate threshold estimated by gas exchange in men and women. Eur J Appl Physiol Occup Physiol 1997, 76(2):157-164.

59. Wasserman K, Hansen JE, Sue DY, Stringer WW, Sietsema KE, Sun XG, Whipp BJ: Principles of exercise testing and interpretation: including pathophysiology and clinical applications. Philadelphia, PA: Lippincott Williams \& Wilkins: 2011.

60. Hansen JE, Casaburi R, Cooper DM, Wasserman K: Oxygen uptake as related to work rate increment during cycle ergometer exercise. Eur J Appl Physiol Occup Physiol 1988, 57(2):140-145.

61. Porszasz J, Casaburi R, Somfay A, Woodhouse LJ, Whipp BJ: A treadmill ramp protocol using simultaneous changes in speed and grade. Med SCi Sports Exerc 2003, 35(9):1596-1603. 
62. Day JR, Rossiter HB, Coats EM, Skasick A, Whipp BJ: The maximally attainable VO2 during exercise in humans: the peak vs. maximum issue. J Appl Physiol 2003, 95(5):1901-1907.

63. Bruce RA, Cooper MN, Gey GO, Fisher LD, Peterson DR: Variations in responses to maximal exercise in health and in cardiovascular disease. Angiology 1973, 24(11):691-702.

64. American Thoracic Society, American College of Chest Physicians: ATS/ ACCP statement on cardiopulmonary exercise testing. Am J Respir Crit Care Med 2003, 167:211-277.

65. Balady GJ, Arena R, Sietsema K, Myers J, Coke L, Fletcher GF, Forman D, Franklin B, Guazzi M, Gulati M, Keteyian SJ, Lavie CJ, MacKo R, Mancini D, Milani RV: Clinician's guide to cardiopulmonary exercise testing in adults: A scientific statement from the American heart association. Circulation 2010, 122(2):191-225.

66. Fletcher GF, Balady GJ, Amsterdam EA, Chaitman B, Eckel R, Fleg J Froelicher VF, Leon AS, Pina IL, Rodney R, Simons-Morton DG, Williams MA, Bazzarre T: Exercise standards for testing and training - a statement for healthcare professionals from the American Heart Association. Circulation 2001, 104(14):1694-1740

67. Garrard CS, Emmons C: The reproducibility of the respiratory responses to maximum exercise. Respiration 1986, 49(2):94-100

68. Nordrehaug JE, Danielsen R, Stangeland L, Rosland GA, Vik-Mo H: Respiratory gas exchange during treadmill exercise testing: reproducibility and comparison of different exercise protocols. Scand J Clin Lab Invest 1991, 51(7):655-658

69. Barron A, Dhutia N, Mayet J, Hughes AD, Francis DP, Wensel R: Test-retest repeatability of cardiopulmonary exercise test variables in patients with cardiac or respiratory disease. Eur J Prev Cardiol 2014, 21(4):445-453.

70. da Cunha IT, Lim PA, Qureshy H, Henson H, Monga T, Protas EJ: Gait outcomes after acute stroke rehabilitation with supported treadmill ambulation training: A randomized controlled pilot study. Arch Phys Med Rehabil 2002, 83(9):1258-1265.

71. Duncan P, Studenski S, Richards L, Gollub S, Lai SM, Reker D, Perera S, Yates J, Koch V, Rigler S, Johnson D: Randomized clinical trial of therapeutic exercise in subacute stroke. Stroke 2003, 34(9):2173-2180.

72. Letombe A, Cornille C, Delahaye H, Khaled A, Morice O, Tomaszewski A, Olivier N: Early post-stroke physical conditioning in hemiplegic patients: a preliminary study. Ann Phys Rehabil Med 2010, 53(10):632-642.

73. Tang A, Sibley KM, Thomas SG, Bayley MT, Richardson D, Mcllroy WE, Brooks D: Effects of an aerobic exercise program on aerobic capacity, spatiotemporal gait parameters, and functional capacity in subacute stroke. Neurorehabil Neural Repair 2009, 23(4):398-406.

74. Katz-Leurer M, Shochina M, Carmeli E, Friedlander Y: The influence of early aerobic training on the functional capacity in patients with cerebrovascular accident at the subacute stage. Arch Phys Med Rehabil 2003, 84(11):1609-1614.

75. Altman DG: Practical Statistics for Medical Research. CRC Press; 1990

76. Roth EJ: Heart disease in patients with stroke: incidence, impact, and implications for rehabilitation. Part 1: Classification and prevalence. Arch Phys Med Rehabil 1993, 74(7):752-760.

doi:10.1186/1743-0003-11-145

Cite this article as: Stoller et al: Cardiopulmonary exercise testing early after stroke using feedback-controlled robotics-assisted treadmill exercise: test-retest reliability and repeatability. Journal of NeuroEngineering and Rehabilitation 2014 11:145.

\section{Submit your next manuscript to BioMed Central and take full advantage of:}

- Convenient online submission

- Thorough peer review

- No space constraints or color figure charges

- Immediate publication on acceptance

- Inclusion in PubMed, CAS, Scopus and Google Scholar

- Research which is freely available for redistribution 